\title{
EXAMES COLPOCITOLÓGICOS NÃO RETIRADOS DE UMA UNIDADE BÁSICA DE SAÚDE DO ACRE
}

Inarrayana Mendonça de Lima ${ }^{1}$, Thatiane Acasio de Almeida Limas ${ }^{1}$, Gullivar

Carvalho da Silva ${ }^{1}$, Rafael Tavares Lima Izel ${ }^{1}$, Eder Ferreira de Arruda ${ }^{2}$

1. Bacharel em Enfermagem pelo Centro Universitário UNINORTE, Rio Branco-AC, Brasil.

2. Docente do Centro Universitário UNINORTE, Rio Branco-AC, Brasil. E-mail: ederarrud@gmail.com.

Recebido em: 15/05/2020 - Aprovado em: 15/06/2020 - Publicado em: 30/06/2020

DOI: 10.18677/EnciBio_2020B28

RESUMO
No Brasil, o câncer do colo do útero se configura como um importante causa de morbimortalidade, embora exista o exame preventivo que é a principal medida para o rastreamento precoce da neoplasia. Este trabalho teve o objetivo de analisar características relacionadas aos exames colpocitológicos não retirados em uma unidade de saúde de Rio Branco-Acre. Tratou-se de um estudo descrito observacional, de corte tipo transversal, com abordagem quantitativa que foi realizado com 44 mulheres por meio da aplicação de um questionário sobre as características sociodemográficas, clínicas e sobre os motivos da não retirada do exame preventivo. Observou-se que a maioria das mulheres estava na faixa etária de 19 a 30 anos $(34,1 \%)$, tinha o ensino médio completo $(61,4 \%)$, exercia atividade remunerada $(84,4 \%)$, com companheiro $(50,0 \%)$ e morava na zona urbana $(93,2 \%)$. Com relação os aspectos clínicos da colpocitologia, a maior parte das participantes tinha sua microbiota uterina colonizada por cocos e Lactobacillus $s p$. (63,6\%), apresentava epitélio escamoso e glandular (59,1\%), com presença de zona de transformação $(68,2 \%)$ e diagnóstico de inflamação $(86,4 \%)$. No que diz respeito aos aspectos relacionados à realização e não retirada do exame, 50,0\% faziam o exame anualmente, $88,6 \%$ consideravam o exame importante, $95,5 \%$ conheciam sua a finalidade, $61,4 \%$ não retiraram o exame por esquecimento e nenhuma mulher relatou ter medo do resultado. Percebe-se que a frequência de exames não retirados é alta e o esquecimento foi o principal motivo apontado para o não retorno à unidade de saúde para o recebimento do resultado do exame colpocitológico.

PALAVRAS-CHAVE: Atenção primária à saúde; Saúde da mulher; Teste de Papanicolau.

\section{COLPOCYTOLOGICAL EXAMS NOT REMOVED FROM A BASIC HEALTH UNIT IN ACRE}

\section{ABSTRACT}

In Brazil, cervical cancer is an important cause of morbidity and mortality, although there is a preventive exam, which is the main measure of early cancer screening. This work had or had as objective to analyze characteristics related to the non-retired colpocytological exams in a health unit in Rio Branco-Acre. This was a described, cross-sectional study, with a quantitative approach that was carried out with 44 
women through the application of a questionnaire on sociodemographic and clinical characteristics and on the reasons for not removing the preventive exam. He observed that the majority of women were in the age group of 19 to 30 years (34.1\%), had completed high school (61.4\%), engaged in paid activity (84.4\%), with a partner $(50,0 \%)$ and lived in the urban area (93.2\%). Regarding the clinical aspects of colpocytology, most participants had their uterine microbiota colonized by coconuts and Lactobacillus sp. (63.6\%), has squamous and glandular epithelium $(59.1 \%)$, with the presence of a transformation zone $(68.2 \%)$ and diagnosis of inflammation (86.4\%). With regard to aspects related to the performance and not withdrawing from the exam, 50.0\% take the exam, $88.6 \%$ consider it an important exam, $95.5 \%$ know its use, $61.4 \%$ do not undergo the exam by forgetfulness and no woman reported being afraid of the result. It is noticed that the frequency of nonretired examinations is high and forgetfulness was the main reason pointed for the return of the health unit to receive results of the Pap smear test.

KEYWORDS: Primary Health Care; Women's Health; Pap test.

\section{INTRODUÇÃO}

O câncer de colo de útero (CCU) tem origem multifatorial, mas a principal causa decorre da infecção por algum tipo de Papiloma Vírus Humano (HPV) que podem ocorrer alterações celulares que podem evoluir para neoplasia cervical (INCA, 2019a).

O exame colpocitológico é o método de rastreamento do CCU e de suas lesões precursoras, sendo indicado para todas as mulheres entre 25 e 64 anos de idade que iniciaram a atividade sexual. Os dois primeiros exames devem ser realizados anualmente, caso os resultados sejam negativos, os próximos devem ser realizado a cada três anos. Sendo excluídas do rastreio mulheres acima de 64 anos de idade, sem história prévia de neoplasias e que tiveram nos últimos cinco anos pelo menos dois exames negativos consecutivos (INCA, 2016).

Apesar de o exame colpocitológico ser um método simples, de baixo custo, de fácil execução e eficaz no diagnóstico precoce, ainda há altas taxas de incidência e de mortalidade por CCU. Em todo o mundo, em 2018, estimaram-se cerca de 570.000 casos de CCU, correspondendo a um risco estimado de 15,1 casos a cada 100 mil mulheres e 311.000 mortes, configurando-se como o quarto tipo de câncer mais diagnosticado e a quarta principal causa de morte por câncer em mulheres (BRAY et al., 2018). No Brasil, entre 2020 e 2022, os casos novos esperados de CCU para cada ano, serão de 16.590 , representando 15,43 casos a cada 100 mil mulheres (INCA, 2019b).

No Brasil, entre os anos de 2016 a 2018, houve um aumento nas coletas citopatológicas do colo do útero, passou de 8.796.344 em 2016 para 8.818.913 coletas em 2018. Embora, exista um aumento na adesão ao rastreio, ainda é elevado o número de mulheres que não retornam as unidades básicas de saúde (UBS) para receberem o resultado do exame (INCA, 2019c).

Neste contexto, o conhecimento sobre os motivos da não retirada do exame de Papanicolau, pode auxiliar no planejamento de ações e atividades, visando o aumento da adesão completa ao exame e melhoria da prevenção e tratamento precoce do CCU. Portanto, este estudo teve como objetivo analisar características relacionadas aos exames colpocitológicos não retirados em uma unidade de saúde de Rio Branco-Acre. 


\section{MATERIAIS E MÉTODOS}

Trata-se de um estudo observacional descritivo, de corte transversal, com abordagem quantitativa. Foi realizado em uma unidade básica de saúde de Rio Branco-AC, Brasil, composta por equipe multidisciplinar completa e que possui uma ampla área de abrangência. A escolha do local se deu em função de se tratar de unidade escola de referência para a instrumentalização da prática de acadêmicos.

O município de Rio Branco-Acre (Latitude: 9॰58'26”S; Longitude: 6748'27"O) está localizado na Amazônia Sul Ocidental, possui uma área territorial de 8.834,942 $\mathrm{km}^{2}$, densidade demográfica estimada em $38,03 \mathrm{hab} . / \mathrm{km}^{2}$ e uma população estimada de 401.155 pessoas (IBGE, 2019).

Foram incluídas mulheres com idade igual ou superior a 18 anos, que realizaram a coleta do exame colpocitológico na unidade e não retornaram para retirá-lo e foram excluídas aquelas que não foram localizadas para a realização da entrevista.

Durante os anos de 2017 e 2018 um total de 479 mulheres realizou coleta para - exame colpocitológico na referida unidade. Dentre estas, 50 pacientes não retornaram para retirar o exame e apresentar o resultado a um profissional de saúde.

Durante os meses de julho e agosto de 2019 foram realizadas ligações telefônicas para as 50 mulheres elegíveis para explicação do objetivo da pesquisa, agendamento da entrevista, assinatura do Termo de Consentimento Livre Esclarecido (TCLE) e entrega do exame no próprio domicílio da paciente. Destas, uma estava abaixo da faixa etária estabelecida e cinco não foram localizadas, assim a amostra de estudo foi composta por 44 mulheres.

Para coleta de dados foi aplicado um questionário com perguntas sobre as características sociodemográficas (faixa etária, escolaridade, situação conjugal, atividade laboral e zona de moradia), os aspectos clínicos da colpocitologia (microbiologia, epitélio uterino, zona de transformação e diagnóstico) e sobre aspectos relacionados ao exame preventivo (frequência, importância, finalidade e barreiras para não retirada).

Os dados foram digitados e revisados no programa Microsoft ${ }^{\circledR}$ Office Excel e analisados no programa estatístico Statistical Package for the Social Sciences (SPSS), versão 21.0, onde foram calculadas as frequências absolutas e relativas para as variáveis de interesse.

A pesquisa foi aprovada pelo Comitê de Ética e Pesquisa do Centro Universitário UNINORTE, com o parecer número 3.000 .295 e CAAE 97746818.3.0000.8028 e ao Comitê de Ética e Pesquisa do Hospital das Clínicas do Acre - HCA/FUNDACRE, com o parecer número 3.054 .092 e CAAE 97746818.3.3001.5009.

\section{RESULTADOS E DISCUSSÃO}

Com base na tabela $1,34,1 \%$ das entrevistadas tinham entre 19 a 30 anos de idade. Diferentemente, no estudo realizado por Rocha et al. (2016) em Belém (PA) a maioria das mulheres tinha mais de 50 anos de idade (41,92\%), somente $10,89 \%$ tinham idade entre 20 e 29 anos. De igual modo, Dantas et al. (2018) ao realizarem uma pesquisa no munícipio de Messias Targino (RN) no intuito de identificar fatores que colaboram para a não adesão ao Papanicolau verificaram que a maior parte das mulheres $(35,0 \%)$ tinha 41 anos ou mais de idade. 
Apesar da maioria dos casos de CCU ocorrer em mulheres acima de 30 anos, a prevenção deve ser iniciada precocemente ainda na fase juvenil, em todas as mulheres entre 25 e 64 anos de idade que iniciaram a atividade sexual, pois o início das atividades sexuais precoce aumenta o risco de CCU (FEBRASCO, 2017). N

No entanto, dificuldades regionais no acesso das mulheres aos serviços de saúde associadas à oferta irregular ou limitada do exame podem influenciar na faixa etária e em outros aspectos relacionados à realização do exame (AGUILAR; SOARES, 2015).

No que se refere à escolaridade, $61,4 \%$ das participantes haviam cursado 0 ensino médio (Tabela 1). De igual modo, no estudo de Navarro et al. (2015) a maior parte das mulheres de Boa Vista $(R R)$ também tinha o ensino médio $(46,0 \%)$.

O nível de escolaridade é um importante fator de prevenção do câncer cérvicouterino, tendo em vista que quanto maior é o nível de instrução melhor é a compreensão das mulheres sobre a importância e necessidade da realização do exame preventivo (CARVALHO et al., 2015).

Tabela 01 - Características sociodemográficas das mulheres que participaram da entrevista de uma unidade básica de saúde da cidade de Rio Branco, Acre, 2019.

\begin{tabular}{llc}
\hline Variável & $\mathbf{N}$ & $\%$ \\
\hline Faixa etária (anos) & 15 & 34,1 \\
$19-30$ & 12 & 27,3 \\
$31-40$ & 07 & 15,9 \\
$41-50$ & 10 & 22,7 \\
$>51$ & & \\
Escolaridade & 07 & 15,9 \\
Ensino fundamental & 27 & 61,4 \\
Ensino médio & 10 & 22,7 \\
Ensino superior & & \\
Situação conjugal & 22 & 50,0 \\
Com companheiro & 22 & 50,0 \\
Sem companheiro & & 13,6 \\
Atividade laboral & 06 & 86,4 \\
Com remuneração & 38 & 93,2 \\
Sem remuneração & & 6,8 \\
Zona de moradia & 41 & $\mathbf{1 0 0 , 0}$ \\
Zona urbana & 03 & $\mathbf{4 4}$ \\
Zona rural & $\mathbf{4 4}$ & \\
\hline Total & & \\
\hline
\end{tabular}

Com relação à atividade laboral, 86,4\% das mulheres não possuíam atividade remunerada (Tabela 1). Da mesma forma, no estudo realizado por Melo et al. (2019) em Recife (PE) foi identificado que 60,2\% das mulheres não tinham ocupação.

Todavia, de acordo com Aguilar e Soares (2015) a inserção das mulheres no mercado de trabalho se constitui como uma importante barreira à realização do exame preventivo, pois o acúmulo da jornada de trabalho com o cuidado familiar pode sobrecarregar a mulher dificultando a compatibilidade de horários entre a atividade laboral e o horário de funcionamento dos serviços de saúde. 
Metade das entrevistadas tinha companheiro (50,0\%), conforme tabela 1. Diferentemente, no estudo realizado por Melo et al. (2017) com 390 mulheres no município de Colorado (PR) no qual evidenciaram que $72,8 \%$ das mulheres eram casadas.

Mulheres com companheiro possuem vida sexual mais ativa, quando comparada as solteiras e viúvas. Portanto, ressalta-se a importância da periodicidade na realização do exame nessa população. Por outro lado, as mulheres solteiras que tenham múltiplos parceiros tem maior risco de ter CCU (ANDRADE et al., 2014).

No que diz respeito ao local de moradia, 93,2\% das mulheres residem na zona urbana (tabela 1). Esse resultado corrobora com os achados de Iglesias et al. (2019) no qual mulheres que residem em zona urbana tem fácil acesso à UBS, sendo fator positivo para realização e retirada do exame para CCU.

Conforme a tabela 2, no que se refere à microbiota uterina, 63,6\% apresentaram cocos e Lactobacillus sp. Segundo Brasil (2013), os achados microbiológicos compatíveis com Lactobacillus sp., cocos e bacilos são considerados achados normais, pois fazem parte da flora vaginal e não caracterizam infecções que necessitem de tratamento.

Tabela 02 - Características colpocitológicas dos exames não retirados em uma unidade básica de saúde da cidade de Rio Branco, Acre, 2019.

\begin{tabular}{lcc}
\hline Variável & $\mathbf{N}$ & $\%$ \\
\hline Microbiologia & & \\
Cândida sp. & 02 & 4,5 \\
Trichomonas vaginalis & 02 & 4,5 \\
Cocos e Lactobacillus sp. & 28 & 63,6 \\
Gardinerela sp. & 08 & 18,2 \\
Outros & 04 & 9,1 \\
Epitélio Uterino & & \\
Escamoso & 11 & 25,0 \\
Escamoso e glandular & 26 & 59,1 \\
Escamoso, glandular e metaplásico & 07 & 15,9 \\
Zona de transformação & & \\
Presente & 30 & 68,2 \\
Ausente & 14 & 21,8 \\
Diagnóstico & & \\
Inflamação & 41 & 93,2 \\
Outros & 03 & 6,8 \\
\hline Total & $\mathbf{4 4}$ & $\mathbf{1 0 0 , 0}$ \\
\hline
\end{tabular}

No que se refere ao epitélio do colo uterino, evidenciou que a maior parte das mulheres apresentou no exame, tecido epitelial escamoso e glandular $(59,1 \%)$ e $68,2 \%$ apresentaram zona de transformação (Tabela 2). De maneira semelhante, no estudo de Gasparin et al. (2016), 75,6\% dos exames apresentaram tecido epitelial escamoso e glandular, como também teve predomínio da presença da zona de transformação nos exames coletados (74,8\%).

A metaplasia escamosa consiste em um processo de transformação do epitélio glandular para epitélio escamoso que ocorre, predominantemente, em mulheres 
mais jovens, tornando-as vulneráveis ao desenvolvimento de lesões intraepiteliais cervicais e ao risco de infecção pelo HPV (FERREIRA et al., 2015).

O início precoce das relações sexuais está associado com o aumento do risco de apresentar câncer cervical e esta relação pode ser explicada com base na consideração de que a zona de transformação do epitélio cervical é mais proliferativa durante a puberdade e a adolescência (período vulnerável), sendo especialmente susceptível a alterações que podem ser induzidas por agentes transmitidos por via sexual, entre eles o HPV (MACHADO et al., 2017).

De acordo com os dados da tabela 2, o principal diagnóstico do exame colpocitológico foi inflamação (86,4\%). Segundo o Brasil (2013), as manifestações inflamatórias e/ou infecciosas do trato genital feminino inferior e do epitélio escamoso do colo uterino são denominadas vulvovaginites e se configuram como alterações comuns nos exames colpocitológico de mulheres brasileiras, sendo a sintomatologia variável de acordo com a etiologia, podendo se manifestar pela presença de corrimento vaginal cujas características podem diferir bastante, sendo capaz de apresentar coloração variada (branca, amarelada, acinzentada, esverdeada), ter ou não odor desagradável, dor, irritação, prurido ou ardência na vagina ou na vulva, dor ou ardor ao urinar e sensação de desconforto pélvico.

Conforme a tabela $3,50,0 \%$ das participantes realizava o exame colpocitológico anualmente. Da mesma forma, na pesquisa realizado por Ferreira et al. (2015) a maioria das mulheres de Aracaju (SE) referiram intervalo de tempo de um ano entre o penúltimo e o último exame (38,4\%). Assim como, no estudo de Silva (2018) no município de Espinosa (MG) no qual 68,1\% das mulheres referiram ter realizado o último exame preventivo no intervalo de 1 a 2 anos.

As mulheres, deste estudo, estão de acordo com as orientações do Ministério da Saúde que preconiza que o exame preventivo deve ser realizado anualmente, mas pode ser feito a cada três anos, desde que a mulher tenha tido resultado negativo para malignidade em dois exames consecutivos (INCA, 2016).

Contudo, segundo Nascimento e Araújo (2014) diversos motivos podem influenciar de forma negativa na periodicidade da realização de exame citopatológico, dentre eles se destacam as motivações relacionadas a sentimentos (vergonha, medo e questões culturais), pouca informação, sobrecarga do cotidiano, dificuldades na relação profissional/usuária e barreiras impostas pelo próprio serviço de saúde.

Tabela 03 - Aspectos relacionados à realização do exame colpocitológico e os motivos da não retirada em uma unidade básica de saúde da cidade de Rio Branco, Acre, 2019.

\begin{tabular}{lcc}
\hline Variável & $\mathbf{N}$ & $\%$ \\
\hline Qual a frequência de realização do exame? & 22 & 50,0 \\
Anualmente & 04 & 9,1 \\
Bianual & 18 & 40,9 \\
Quando considera necessário & & \\
Considera o exame importante? & 39 & 88,6 \\
Sim & 05 & 11,4 \\
Não & & \\
Conhece a finalidade do exame? & 42 & 95,5 \\
Sim & 02 & 4,5
\end{tabular}


Motivos da não retirada do exame

\begin{tabular}{lcc} 
Esquecimento & 27 & 61,4 \\
Indisponibilidade de tempo & 14 & 31,8 \\
Distância da unidade & 03 & 6,8 \\
Medo do resultado? & & \\
Não & 44 & 100 \\
\hline Total & 44 & $\mathbf{1 0 0 , 0}$ \\
\hline
\end{tabular}

Dentre as entrevistadas, 88,6\% consideraram importante à realização e $95,5 \%$ referiram conhecer a finalidade do exame colpocitológico. De forma similar, no estudo realizado por Vasconcelos et al. (2017) em Carmo da Mata (MG) foi observado que $93,0 \%$ das mulheres tinham conhecimento adequado sobre o exame e $91,0 \%$ consideraram o exame necessário.

De acordo com Melo et al. (2019) o conhecimento e a percepção da importância do exame colpocitológico é determinante no comportamento pessoal e influencia na adesão das mulheres as medidas preventivas do CCU.

Apesar das mulheres, do presente estudo, terem referido conhecimento préexistente sobre a finalidade e importância do exame preventivo, é necessário trabalhar constantemente a educação em saúde para prevenir o CCU (SOUZA et al., 2015).

Dentre os motivos para não retirada do exame colpocitológico, $61,4 \%$ das participantes relataram esquecimento e nenhuma mulher mencionou ter tido medo do resultado (Tabela 3). Já na pesquisa realizada por Azevedo et al. (2016) no município de Brejo do Cruz (PB) foi identificado que os principais fatores impeditivos para o não retorno à unidade para retirada do exame era devido terem vergonha, falta de tempo e medo do resultado.

Há muitas barreiras frente à prevenção do CCU, que impede a ida ou retorno das mulheres as UBS para realização, retirada e apresentação do exame. Muitas mulheres têm vergonha e são tímidas em relação à exposição do órgão sexual para realizar o procedimento, há o pensamento de invasão da privacidade e integridade corporal, como também uma experiência dolorosa e desagradável. Muitas mulheres desconhecem a finalidade do exame, pensando que é pra detectar doenças sexualmente transmissíveis (IST), procurando fazer o exame, somente quando tiver algum sinal e sintomas (MONTEIRO et al., 2017).

Neste contexto, as unidades de atenção básica devem adotar uma postura acolhedora por meio da construção de vínculo entre profissional/usuária, permitindo práticas educativas em saúde mais eficientes e que favoreçam a superação das barreiras enfrentadas pelas mulheres para a realização do exame preventivo e posterior retorno ao serviço de saúde (MELO et al., 2019).

\section{CONCLUSÕES}

A frequência de exames não retirados no período analisado foi alta e o esquecimento foi o principal motivo apontado para o não retorno a UBS para 0 recebimento do resultado do exame colpocitológico. Fato este que pode trazer prejuízo para a saúde da mulher, tais como: não ter acesso a informações necessárias, não realizar o diagnóstico e tratamento precoce e não ser encaminhada a repetição do exame, caso necessário.

Todavia, é necessário considerar que o viés de memória das participantes ao responder as questões sobre os aspectos relacionados à realização do exame 
preventivo tenha sido a principal limitação do estudo. Porém, os achados permitem sugerir que exista a necessidade de realização de busca ativa das mulheres que não retornam para retirar o referido exame, bem como a ampliação das campanhas sobre a importância do exame, principalmente, a disseminação da informação de que o diagnóstico precoce de lesões aumenta as chances de cura do CCU.

\section{REFERÊNCIAS}

AGUILAR, R. P.; SOARES, D. A. Barreiras à realização do exame Papanicolau: perspectivas de usuárias e profissionais da estratégia de saúde da família da cidade de Vitória da Conquista-BA. Revista de Saúde Coletiva, v. 25, n.2, p.359-379, 2015. Doi: https://doi.org/10.1590/S0103-73312015000200003.

ANDRADE, A.K; ALMEIDA, M.M.G., ARAUJO, T.M.; SANTOS, K.O.B. Fatores associados a não adesão ao Papanicolau entre mulheres atendidas pela Estratégia Saúde da Família em Feira de Santana, Bahia, 2010. Epidemiologia e Serviços de Saúde, v.23, n.1, p.111-120, 2014. Doi: https://doi.org/10.5123/S167949742014000100011.

AZEVEDO, A.G., CAVALCANTE, I.B., CAVALCANTE, J.B.; ROLIM, L.A.L.D.M.M. Fatores que influenciam a não realização do exame papanicolau e o impacto de ações educativas. Revista Brasileira de Análises Clínicas, v. 48, n.3, p. 32-43, 2016. Disponível em: <http://www.rbac.org.br/artigos/fatores-que-influenciam-a-naorealizacao-do-exame-de-papanicolaou-e-o-impacto-de-acoes-educativas-48n-3/>

BRASIL. Ministério da Saúde. Controle dos cânceres do colo do útero e de mama. Brasília: Editora do Ministério da Saúde, 2013. 124p. Disponível em: < http://bvsms.saude.gov.br/bvs/publicacoes/controle_canceres_colo_utero_2013.pdf>

BRAY, F.; FERLAY, J.; SOERJOMATARAM, I.; SIEGEL, R.L.; TORRE, L.A. et al. Global Cancer Statistics 2018: GLOBOCAN Estimates of Incidence and Mortality Worldwide for 36 Cancers in 185 Countries. CA: A Câncer Journal for Clinicians, v.68, p.394-424, 2018. Doi: https://doi.org/10.3322/caac.21492.

CARVALHO, B.A.; SILVA, J.C.M.; FALAVIGNA, M.F.; SILVA, M.F.; FRAZILLI, R.T.V. Exame Papanicolau: percepção de acadêmicas de enfermagem do Vale do Paraíba. Revista Eletrônica de Enfermagem do Vale do Paraíba, v.1, n.8, p.43-62, 2015. Disponível

em: <http://unifatea.com.br/seer3/index.php/REENVAP/article/download/38/27/>.

DANTAS, P.V.J.; LEITE, K.N.S.; CÉSAR, E.S.R.; SILVA, S.C.R.; SOUZA, E.A. et al. Conhecimento das mulheres e fatores da não adesão acerca do exame papanicolau. Revista de enfermagem UFPE on line, v.12, n.3, p.684-691, 2018. Disponível em: <https://periodicos.ufpe.br/revistas/revistaenfermagem/article/download/22582/2806>

FEDERAÇÃO BRASILEIRA DAS ASSOCIAÇÕES DE GINECOLOGIA E OBSTETRÍCIA. Rastreio, diagnóstico e tratamento do câncer de colo de útero. São Paulo: FEBRASGO, $2017 . \quad$ Disponível em: <https://www.febrasgo.org.br/media/k2/attachments/05Z- 
ZDIAGNOYSTICOZRASTREIOZEZTRATAMENTOZDOZCAyNCERZDEZCOLOZDE ZUYTERO.pdf>.

FERREIRA, J.E.L.; ALVES, M.C.; MARTINS, M.C.V.; ROSA, N.P.R.S.; GONÇAVES, M.C. Perfil da população atendida em um consultório de atendimento integral à saúde da mulher. Revista Ciências Biológicas e de Saúde, v.3, n.1, p.127-140, $2015 . \quad$ Disponível em: $<$ https://periodicos.set.edu.br/index.php/cadernobiologicas/article/view/2490/1462>

GASPARIN, V.A.; PITILIN, E.B.; METESKI, F.K.; GEREMIA, D.S.; SIVA FILHO, C.C. Fatores associados à representatividade da zona de transformação em exames citopatológicos do colo uterino. Revista Cogitare Enfermagem, v. 21, n. 2, 2016. Doi: https://dx.doi.org/10.5380/ce.v21i2.44241

INSTITUTO BRASILEIRO DE GEOGRAFIA E ESTATÍSTICA. Rio Branco (AC). 2019. Disponível em: <https://cidades.ibge.gov.br/brasil/ac/rio-branco/panorama>

IGLESIAS, G.A.; LARRUBIA, L.G.; CAMPOS NETO, A.S.; PACCA, F.C.; IEMBO, T. Conhecimento e adesão ao Papanicolau de mulheres de uma rede de Atenção Primária à Saúde. Revista de Ciências Médicas, v. 28, n. 1, p. 21-30, 2019. Doi: https://dx.doi.org/10.24220/2318-0897v28n1a4008

INSTITUTO NACIONAL DE CÂNCER. Diretrizes brasileiras para o rastreamento do câncer do colo do útero. Rio de Janeiro: INCA, 2016. Disponível em: https://www.inca.gov.br/sites/ufu.sti.inca.local/files//media/document//diretrizesparaor astreamentodocancerdocolodoutero_2016_corrigido.pdf.

INSTITUTO NACIONAL DE CÂNCER. Tipos de câncer. Rio de Janeiro: INCA, 2019a. Disponível em: <https://www.inca.gov.br/tipos-de-cancer>.

INSTITUTO NACIONAL DE CÂNCER. Estimativa 2020: Incidência de câncer no Brasil. Rio de Janeiro: INCA, 2019b. 120p. Disponível em: $<$ https://www.inca.gov.br/sites/ufu.sti.inca.local/files//media/document//estimativa2020-incidencia-de-cancer-no-brasil.pdf>

INSTITUTO NACIONAL DE CÂNCER. Informativo detecção precoce. Rio de Janeiro: INCA, 2019c. Disponível em: $<$ https://www.inca.gov.br/sites/ufu.sti.inca.local/files//media/document//informativodeteccao-precoce-numero2-2019.pdf>. Acesso em: 24 mar. 2020.

MACHADO, H.S.; SOUZA, M.C.; GONÇALVES, S.J.C. Câncer de colo de útero: análise Epidemiológica e Citopatológica no município de Vassouras-RJ. Revista Pró-UniverSUS, v.8, n.1, p.55-61, 2017. Disponível em: $<$ http://editora.universidadedevassouras.edu.br/index.php/RPU/article/view/904>.

MELO, E. M. F. et al. Câncer cérvico-uterino: conhecimento, atitude e prática sobre o exame de prevenção. Revista Brasileira de Enfermagem, v.72, supl.3, p. 30-36, 2019. Doi: https://doi.org/10.1590/0034-7167-2017-0645 
MELO, W.A.; PELLOSO, S.M.; ALVARENGA, A.; CARVALHO, M.D.B. Fatores associados a alterações do exame citopatológico cérvico-uterino no Sul do Brasil. Revista Brasileira de Saúde Materno Infantil, v. 17, n. 4, p. 654-652, 2017. Doi: http://dx.doi.org/10.1590/1806-93042017000400002.

MONTEIRO, N.O.; ALVES, I.S.P.; ROCHA, L.S. Fatores associados a não realização rotineira do exame Papanicolau pelas mulheres. Revista Cientifica Univiçosa, v.9, n.1, p.651-656, 2017. Disponível em: $<$ https://academico.univicosa.com.br/revista/index.php/RevistaSimpac/article/view/88 6/998>

NASCIMENTO, R. G.; ARAÚJO, A. Falta de periodicidade na realização do exame citopatológico do colo uterino: motivações das mulheres. Revista Mineira de Enfermagem, v.18, n.3, p.557-564, 2014. Doi: https://doi.org/ 10.5935/14152762.20140041.

NAVARRO, C.; FONSECA, A.J.; SIBAJEV, A. SOUZA, C.I.A.; ARAÚJO, D.S. et al. Cobertura do rastreamento do câncer de colo de útero em região de alta incidência. Revista de Saúde Pública, v.49, n.17, p.1-8, 2015. Doi: https://doi.org/10.1590/S0034-8910.2015049005554.

ROCHA, S.M.M.; BAHIA, M.O.; ROCHA, C.A.M. Perfil dos exames citopatológico do colo do útero realizados na Casa da Mulher, Estado do Pará, Brasil. Revista PanAmazônica de Saúde, v.7, n.3, p. 51-55, 2016. Doi: http://dx.doi.org/10.5123/S217662232016000300006 .

SILVA, P. L. N. Perfil epidemiológico, clínico e laboratorial do exame citopatológico realizado em Espinosa, Minas Gerais, durante o ano de 2014. Revista Sustinere, v.6, n.2, p.239-249, 2018. Doi: https://doi.org/10.12957/sustinere.2018.32949.

SOUZA, K. R. et al. Educação popular como instrumento participativo paraa prevenção do câncer ginecológico: percepção de mulheres. Revista Cuidarte, v.6, n.1, p.492-499, 2015. Doi: https://dx.doi.org/10.15649/cuidarte.v6i1.129.

VASCONCELOS, L.C.; BUENO, D.S.; SILVA, J.S.M.; RIOS, R.F.D.; PESSOA, R.A. et al. Conhecimento de mulheres a respeito do exame Papanicolau. UNICIÊNCIAS, v.21, n.2, p.105-109, 2017. Doi: https://doi.org/10.17921/1415-5141.2017v21n2p105109. 